\title{
An Efficient Approach for Fractional Harry Dym Equation by Using Sumudu Transform
}

\author{
Devendra Kumar, ${ }^{1}$ Jagdev Singh, ${ }^{2}$ and A. Kılıçman ${ }^{3}$ \\ ${ }^{1}$ Department of Mathematics, JaganNath Gupta Institute of Engineering and Technology, Jaipur, Rajasthan 302022, India \\ ${ }^{2}$ Department of Mathematics, JaganNath University, Village-Rampura, Tehsil-Chaksu, Jaipur, Rajasthan 303901, India \\ ${ }^{3}$ Department of Mathematics and Institute for Mathematical Research, Universiti Putra Malaysia, 43400 Serdang, Selangor, Malaysia
}

Correspondence should be addressed to A. Kilıçman; akilicman@putra.upm.edu.my

Received 13 March 2013; Accepted 22 April 2013

Academic Editor: Mustafa Bayram

Copyright (C) 2013 Devendra Kumar et al. This is an open access article distributed under the Creative Commons Attribution License, which permits unrestricted use, distribution, and reproduction in any medium, provided the original work is properly cited.

An efficient approach based on homotopy perturbation method by using sumudu transform is proposed to solve nonlinear fractional Harry Dym equation. This method is called homotopy perturbation sumudu transform (HPSTM). Furthermore, the same problem is solved by Adomian decomposition method (ADM). The results obtained by the two methods are in agreement, and, hence, this technique may be considered an alternative and efficient method for finding approximate solutions of both linear and nonlinear fractional differential equations. The HPSTM is a combined form of sumudu transform, homotopy perturbation method, and He's polynomials. The nonlinear terms can be easily handled by the use of He's polynomials. The numerical solutions obtained by the HPSTM show that the approach is easy to implement and computationally very attractive.

\section{Introduction}

Fractional differential equations have gained importance and popularity, mainly due to its demonstrated applications in science and engineering. For example, these equations are increasingly used to model problems in fluid mechanics, acoustics, biology, electromagnetism, diffusion, signal processing, and many other physical processes. The most important advantage of using fractional differential equations in these and other applications is their nonlocal property. It is well known that the integer order differential operator is a local operator but the fractional order differential operator is nonlocal. This means that the next state of a system depends not only upon its current state but also upon all of its historical states. This is more realistic and it is one reason why fractional calculus has become more and more popular [1-9].

In this paper, we consider the following nonlinear timefractional Harry Dym equation of the form

$$
D_{t}^{\alpha} U(x, t)=U^{3}(x, t) D_{x}^{3} U(x, t), \quad 0<\alpha \leq 1,
$$

with the initial condition

$$
U(x, 0)=\left(a-\frac{3 \sqrt{b}}{2} x\right)^{2 / 3},
$$

where $\alpha$ is parameter describing the order of the fractional derivative and $U(x, t)$ is a function of $x$ and $t$. The fractional derivative is understood in the Caputo sense. The general response expression contains a parameter describing the order of the fractional derivative that can be varied to obtain various responses. In the case of $\alpha=1$, the fractional Harry Dym equation reduces to the classical nonlinear Harry Dym equation. The exact solution of the Harry Dym equation is given by $[10]$

$$
U(x, t)=\left(a-\frac{3 \sqrt{b}}{2}(x+b t)\right)^{2 / 3},
$$

where $a$ and $b$ are suitable constants. The Harry Dym is an important dynamical equation which finds applications 
in several physical systems. The Harry Dym equation first appeared in Kruskal and Moser [11] and is attributed in an unpublished paper by Harry Dym in 1973-1974. It represents a system in which dispersion and nonlinearity are coupled together. Harry Dym is a completely integrable nonlinear evolution equation. The Harry Dym equation is very interesting because it obeys an infinite number of conversion laws; it does not posses, the Painleve property. The Harry Dym equation has strong links to the Korteweg-de Vries equation, and applications of this equation were found to the problems of hydrodynamics [12]. The Lax pair of the Harry Dym equation is associated with the Sturm-Liouville operator. The Liouville transformation transforms this operator spectrally into the Schrödinger operator [13]. Recently, a fractional model of Harry Dym equation was studied by Kumar et al. [14], and approximate analytical solution was obtained by using homotopy perturbation method (HPM).

In the present paper, the homotopy perturbation sumudu transform method (HPSTM) basically illustrates how the sumudu transform can be used to approximate the solutions of the linear and nonlinear fractional differential equations by manipulating the homotopy perturbation method. The homotopy perturbation method (HPM) was first introduced and developed by He [15-17]. The HPM was also studied by many authors to handle linear and nonlinear equations arising in various scientific and technological fields [1824]. The homotopy perturbation sumudu transform method (HPSTM) is a combination of sumudu transform method, HPM, and He's polynomials and is mainly due to Ghorbani $[25,26]$. In recent years, many authors have paid attention to study the solutions of linear and nonlinear partial differential equations by using various methods combined with the Laplace transform [27-30] and sumudu transform [31, 32].

In this paper, we apply the homotopy perturbation sumudu transform method (HPSTM) and Adomian decomposition method (ADM) to solve the nonlinear timefractional Harry Dym equation. The objective of the present paper is to extend the application of the HPSTM to obtain analytic and approximate solutions to the nonlinear timefractional Harry Dym equation. The advantage of the HPSTM is its capability of combining two powerful methods for obtaining exact and approximate analytical solutions for nonlinear equations. It provides the solutions in terms of convergent series with easily computable components in a direct way without using linearization, perturbation, or restrictive assumptions. It is worth mentioning that the HPSTM is capable of reducing the volume of the computational work as compared to the classical methods while still maintaining the high accuracy of the numerical result; the size reduction amounts to an improvement of the performance of the approach.

\section{Sumudu Transform}

In early 1990s, Watugala [33] introduced a new integral transform, named the sumudu transform and applied it to the solution of ordinary differential equation in control engineering problems. The sumudu transform is defined over the set of functions

$$
\begin{aligned}
A=\{ & f(t) \mid \exists M, \tau_{1}, \tau_{2}>0, \\
& \left.|f(t)|<M e^{|t| \mid \tau_{j}}, \text { if } t \in(-1)^{j} \times[0, \infty)\right\}
\end{aligned}
$$

by the following formula:

$$
\bar{f}(u)=S[f(t)]=\int_{0}^{\infty} f(u t) e^{-t} d t, \quad u \in\left(-\tau_{1}, \tau_{2}\right) .
$$

Some of the properties were established by Weerakoon in [34, 35]. Furthermore, fundamental properties of this transform were also established by Asiru [36]. This transform was applied to the one-dimensional neutron transport equation in [37] by Kadem. In fact it was shown that there is strong relationship between sumudu and other integral transform methods; see Kilıçman et al. [38]. In particular the relation between sumudu transform and Laplace transforms was proved in Kılıçman and Gadain [39]. Next, in Eltayeb et al. [40], the sumudu transform was extended to the distributions and some of their properties were also studied in Kilıçman and Eltayeb [41]. Recently, this transform is applied to solve the system of differential equations; see K1lıçman et al. [42]. Note that a very interesting fact about sumudu transform is that the original function and its sumudu transform have the same Taylor coefficients except for the factor $n$; see Zhang [43]. Thus, if $f(t)=\sum_{n=0}^{\infty} a_{n} t^{n}$, then $\bar{f}(u)=\sum_{n=0}^{\infty} n ! a_{n} u^{n}$; see Kilıçman et al. [38]. Similarly, the sumudu transform sends combinations, $C(m, n)$, into permutations, $P(m, n)$, and, hence, it will be useful in the discrete systems.

\section{Basic Definitions of Fractional Calculus}

In this section, we mention the following basic definitions of fractional calculus.

Definition 1. The Riemann-Liouville fractional integral operator of order $\alpha>0$, of a function $f(t) \in C_{\mu}, \mu \geq-1$, is defined as [3]

$$
\begin{gathered}
J^{\alpha} f(t)=\frac{1}{\Gamma(\alpha)} \int_{0}^{t}(t-\tau)^{\alpha-1} f(\tau) d \tau \quad(\alpha>0), \\
J^{0} f(t)=f(t) .
\end{gathered}
$$

For the Riemann-Liouville fractional integral, we have

$$
J^{\alpha} t^{\gamma}=\frac{\Gamma(\gamma+1)}{\Gamma(\gamma+\alpha+1)} t^{\alpha+\gamma}
$$

Definition 2. The fractional derivative of $f(t)$ in the Caputo sense is defined as [6]

$$
\begin{aligned}
D_{t}^{\alpha} f(t) & =J^{m-\alpha} D^{n} f(t) \\
& =\frac{1}{\Gamma(n-\alpha)} \int_{0}^{t}(t-\tau)^{m-\alpha-1} f^{(m)}(\tau) d \tau,
\end{aligned}
$$

for $m-1<\alpha \leq m, m \in N, t>0$. 
For the Riemann-Liouville fractional integral and the Caputo fractional derivative, we have the following relation:

$$
J_{t}^{\alpha} D_{t}^{\alpha} f(t)=f(t)-\sum_{k=0}^{m-1} f^{(k)}(0+) \frac{t^{k}}{k !}
$$

Definition 3. The sumudu transform of the Caputo fractional derivative is defined as follows [44]:

$$
\begin{aligned}
S\left[D_{t}^{\alpha} f(t)\right]= & u^{-\alpha} S[f(t)] \\
& -\sum_{k=0}^{m} u^{-\alpha+k} f^{(k)}(0+), \quad(m-1<\alpha \leq m) .
\end{aligned}
$$

\section{Solution by Homotopy Perturbation Sumudu Transform Method (HPSTM)}

4.1. Basic Idea of HPSTM. To illustrate the basic idea of this method, we consider a general fractional nonlinear nonhomogeneous partial differential equation with the initial condition of the form

$$
\begin{gathered}
D_{t}^{\alpha} U(x, t)+R U(x, t)+N U(x, t)=g(x, t), \\
U(x, 0)=f(x),
\end{gathered}
$$

where $D_{t}^{\alpha} U(x, t)$ is the Caputo fractional derivative of the function $U(x, t), R$ is the linear differential operator, $N$ represents the general nonlinear differential operator and $g(x, t)$ is the source term.

Applying the sumudu transform (denoted in this paper by $S$ ) on both sides of (11), we get

$$
S\left[D_{t}^{\alpha} U(x, t)\right]+S[R U(x, t)]+S[N U(x, t)]=S[g(x, t)] .
$$

Using the property of the sumudu transform, we have

$$
\begin{aligned}
S[U(x, t)]= & f(x)+u^{\alpha} S[g(x, t)] \\
& -u^{\alpha} S[R U(x, t)+N U(x, t)] .
\end{aligned}
$$

Operating with the sumudu inverse on both sides of (14) gives

$$
U(x, t)=G(x, t)-S^{-1}\left[u^{\alpha} S[R U(x, t)+N U(x, t)]\right]
$$

where $G(x, t)$ represents the term arising from the source term and the prescribed initial conditions. Now we apply the HPM

$$
U(x, t)=\sum_{n=0}^{\infty} p^{n} U_{n}(x, t)
$$

and the nonlinear term can be decomposed as

$$
N U(x, t)=\sum_{n=0}^{\infty} p^{n} H_{n}(U)
$$

for some He's polynomials $H_{n}(U)[26,45]$ that are given by

$$
\begin{array}{r}
H_{n}\left(U_{0}, U_{1}, \ldots, U_{n}\right)=\frac{1}{n !} \frac{\partial^{n}}{\partial p^{n}}\left[N\left(\sum_{i=0}^{\infty} p^{i} U_{i}\right)\right]_{p=0}, \\
n=0,1,2, \ldots
\end{array}
$$

Substituting (16) and (17) in (15), we get

$$
\begin{aligned}
\sum_{n=0}^{\infty} p^{n} U_{n}(x, t) & \\
= & G(x, t) \\
& \quad-p\left(S^{-1}\left[u^{\alpha} S\left[R \sum_{n=0}^{\infty} p^{n} U_{n}(x, t)+\sum_{n=0}^{\infty} p^{n} H_{n}(U)\right]\right]\right),
\end{aligned}
$$

which is the coupling of the sumudu transform and the HPM using He's polynomials. Comparing the coefficients of like powers of $p$, the following approximations are obtained:

$$
\begin{aligned}
& p^{0}: U_{0}(x, t)=G(x, t), \\
& p^{1}: U_{1}(x, t)=-S^{-1}\left[u^{\alpha} S\left[R U_{0}(x, t)+H_{0}(U)\right]\right], \\
& p^{2}: U_{2}(x, t)=-S^{-1}\left[u^{\alpha} S\left[R U_{1}(x, t)+H_{1}(U)\right]\right], \\
& p^{3}: U_{3}(x, t)=-S^{-1}\left[u^{\alpha} S\left[R U_{2}(x, t)+H_{2}(U)\right]\right],
\end{aligned}
$$

Proceeding in this same manner, the rest of the components $U_{n}(x, t)$ can be completely obtained and the series solution is thus entirely determined. Finally, we approximate the analytical solution $U(x, t)$ by truncated series

$$
U(x, t)=\lim _{N \rightarrow \infty} \sum_{n=0}^{N} U_{n}(x, t) .
$$

The previous series solutions generally converge very rapidly. A classical approach of convergence of this type of series is already presented by Abbaoui and Cherruault [46].

4.2. Solution of the Problem. Consider the following nonlinear time-fractional Harry Dym equation:

$$
D_{t}^{\alpha} U(x, t)=U^{3}(x, t) D_{x}^{3} U(x, t), \quad 0<\alpha \leq 1,
$$

with the initial condition

$$
U(x, 0)=\left(a-\frac{3 \sqrt{b}}{2} x\right)^{2 / 3}
$$

Applying the sumudu transform on both sides of (22), subject to initial condition (23), we have

$$
S[U(x, t)]=\left(a-\frac{3 \sqrt{b}}{2} x\right)^{2 / 3}+u^{\alpha} S\left[U^{3}(x, t) D_{x}^{3} U(x, t)\right] .
$$


The inverse Sumudu transform implies that

$$
\begin{aligned}
U(x, t)= & \left(a-\frac{3 \sqrt{b}}{2} x\right)^{2 / 3} \\
& +S^{-1}\left[u^{\alpha} S\left[U^{3}(x, t) D_{x}^{3} U(x, t)\right]\right] .
\end{aligned}
$$

Now applying the HPM, we get

$$
\begin{aligned}
\sum_{n=0}^{\infty} p^{n} U_{n}(x, t)= & \left(a-\frac{3 \sqrt{b}}{2} x\right)^{2 / 3} \\
& +p\left(S^{-1}\left[u^{\alpha} S\left[\sum_{n=0}^{\infty} p^{n} H_{n}(U)\right]\right]\right)
\end{aligned}
$$

where $H_{n}(U)$ are He's polynomials that represent the nonlinear terms. So, the He's polynomials are given by

$$
\sum_{n=0}^{\infty} p^{n} H_{n}(U)=U^{3} D_{x}^{3} U .
$$

The first few components of He's polynomials are given by

$$
\begin{aligned}
& H_{0}(U)=U_{0}^{3} D_{x}^{3} U_{0}, \\
& H_{1}(U)=U_{0}^{3} D_{x}^{3} U_{1}+3 U_{0}^{2} U_{1} D_{x}^{3} U_{0}, \\
& H_{2}(U)=U_{0}^{3} D_{x}^{3} U_{2}+3 U_{0}^{2} U_{1} D_{x}^{3} U_{1}+\left(3 U_{0} U_{1}^{2}+3 U_{0}^{2} U_{2}\right) D_{x}^{3} U_{0},
\end{aligned}
$$

Comparing the coefficients of like powers of $p$, we have

$$
\begin{aligned}
p^{0}: U_{0}(x, t) & =\left(a-\frac{3 \sqrt{b}}{2} x\right)^{2 / 3}, \\
p^{1}: U_{1}(x, t) & =S^{-1}\left[u^{\alpha} S\left[H_{0}(U)\right]\right] \\
& =-b^{3 / 2}\left(a-\frac{3 \sqrt{b}}{2} x\right)^{-1 / 3} \frac{t^{\alpha}}{\Gamma(\alpha+1)}, \\
p^{2}: U_{2}(x, t)= & S^{-1}\left[u^{\alpha} S\left[H_{1}(U)\right]\right] \\
= & -\frac{b^{3}}{2}\left(a-\frac{3 \sqrt{b}}{2} x\right)^{-4 / 3} \frac{t^{2 \alpha}}{\Gamma(2 \alpha+1)}, \\
p^{3}: U_{3}(x, t)= & S^{-1}\left[u^{\alpha} S\left[H_{2}(U)\right]\right] \\
= & b^{9 / 2}\left(a-\frac{3 \sqrt{b}}{2} x\right)^{-7 / 3} \\
& \times\left(\frac{15}{2} \frac{\Gamma(2 \alpha+1)}{2(\Gamma(\alpha+1))^{2}}-16\right) \frac{t^{3 \alpha}}{\Gamma(3 \alpha+1)}
\end{aligned}
$$

In this manner the rest of components of the HPSTM solution can be obtained. Thus, the solution $U(x, t)$ of the (22) is given as

$$
\begin{aligned}
U(x, t)= & \left(a-\frac{3 \sqrt{b}}{2} x\right)^{2 / 3}-b^{3 / 2}\left(a-\frac{3 \sqrt{b}}{2} x\right)^{-1 / 3} \frac{t^{\alpha}}{\Gamma(\alpha+1)} \\
& -\frac{b^{3}}{2}\left(a-\frac{3 \sqrt{b}}{2} x\right)^{-4 / 3} \frac{t^{2 \alpha}}{\Gamma(2 \alpha+1)} \\
& +b^{9 / 2}\left(a-\frac{3 \sqrt{b}}{2} x\right)^{-7 / 3} \\
& \times\left(\frac{15}{2} \frac{\Gamma(2 \alpha+1)}{2(\Gamma(\alpha+1))^{2}}-16\right) \frac{t^{3 \alpha}}{\Gamma(3 \alpha+1)}+\cdots
\end{aligned}
$$

The series solution converges very rapidly. The rapid convergence means that only few terms are required to get analytic function. Now, we calculate numerical results of the approximate solution $U(x, t)$ for different values of $\alpha=1 / 3,1 / 2,1$ and for various values of $t$ and $x$. The numerical results for the approximate solution obtained by using HPSTM and the exact solution given by Mokhtari [10] for constant values of $a=4$ and $b=1$ for various values of $t, x$, and $\alpha$ are shown in Figures 1(a)-1(d), and those for different values of $x$ and $\alpha$ at $t=1$ are depicted in Figure 2. It is observed from Figures $1(\mathrm{a})-1(\mathrm{c})$ that $U(x, t)$ decreases with the increase in both $x$ and $t$ for $\alpha=1 / 3,1 / 2$, and $\alpha=1$. Figures $1(\mathrm{c})-1(\mathrm{~d})$ clearly shows that, when $\alpha=1$, the approximate solution obtained by the HPSTM is very near to the exact solution. It is also seen from Figure 2 that as the value of $\alpha$ increases, the displacement $U(x, t)$ increases. It is to be noted that only the third order term of the HPSTM was used in evaluating the approximate solutions for Figure 1. It is evident that the efficiency of the present method can be dramatically enhanced by computing further terms of $U(x, t)$ when the HPSTM is used.

\section{Solution by Adomian Decomposition Method (ADM)}

5.1. Basic Idea of ADM. To illustrate the basic idea of Adomian decomposition method $[47,48]$, we consider a general fractional nonlinear nonhomogeneous partial differential equation with the initial condition of the form

$$
D_{t}^{\alpha} U(x, t)+R U(x, t)+N U(x, t)=g(x, t),
$$

where $D_{t}^{\alpha} U(x, t)$ is the Caputo fractional derivative of the function $U(x, t), R$ is the linear differential operator, $N$ represents the general nonlinear differential operator, and $g(x, t)$ is the source term.

Applying the operator $J_{t}^{\alpha}$ on both sides of (31) and using result (9), we have

$$
\begin{aligned}
U(x, t)= & \sum_{k=0}^{m-1}\left(\frac{\partial^{k} U}{\partial t^{k}}\right)_{t=0} \frac{t^{k}}{k !}+J_{t}^{\alpha} g(x, t) \\
& -J_{t}^{\alpha}[R U(x, t)+N U(x, t)] .
\end{aligned}
$$




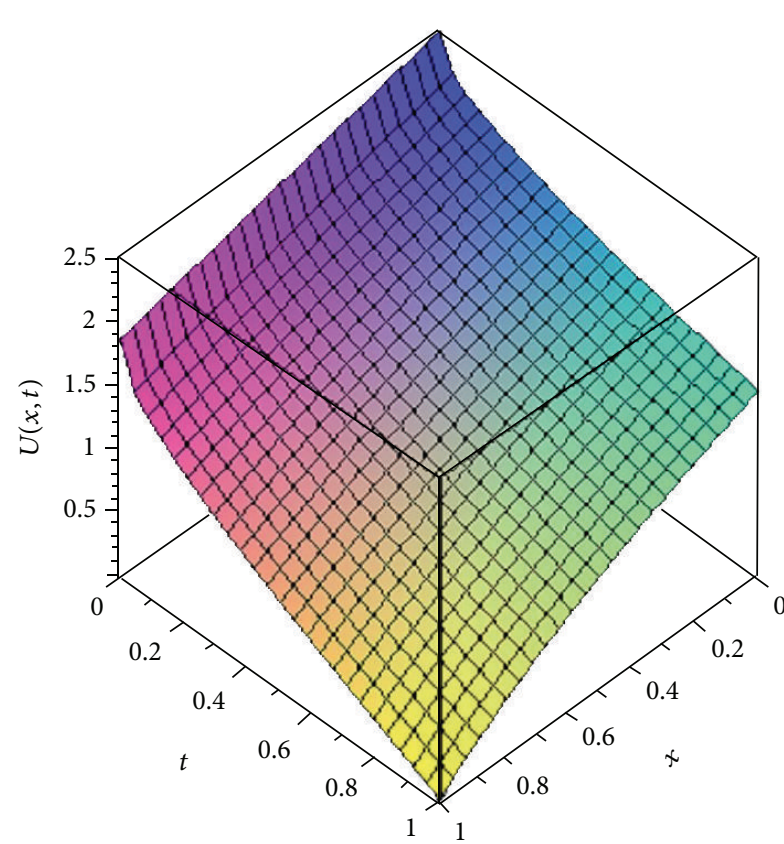

(a)

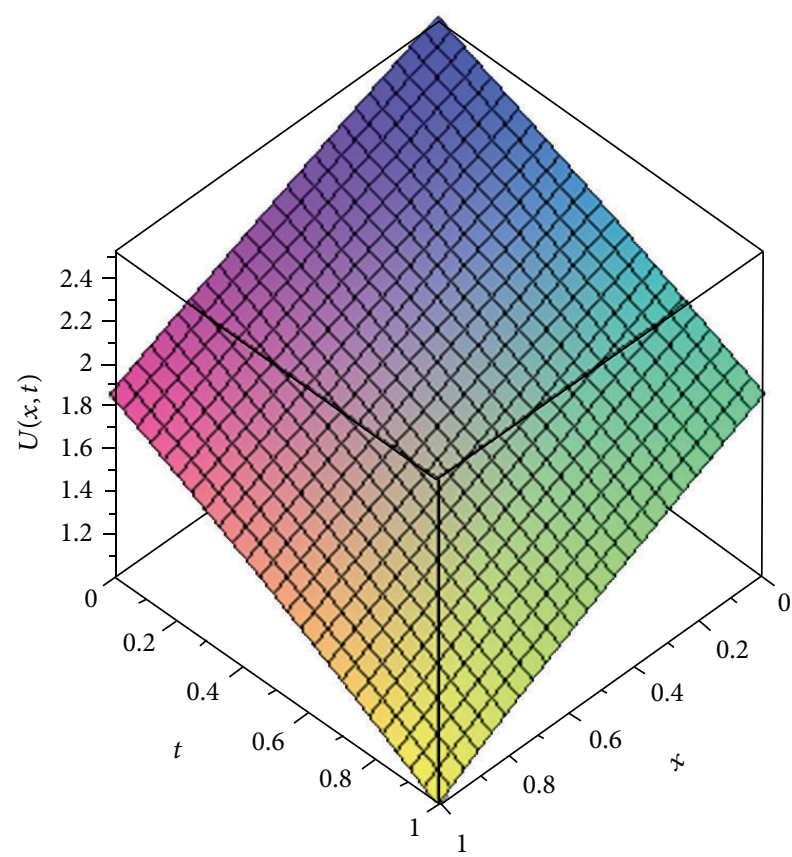

(c)

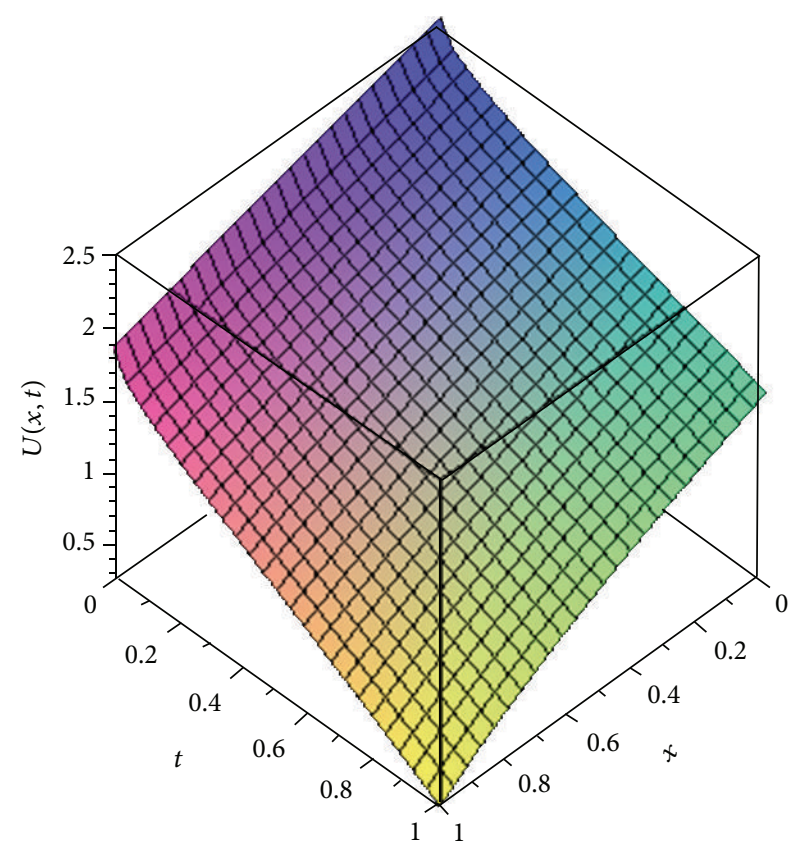

(b)

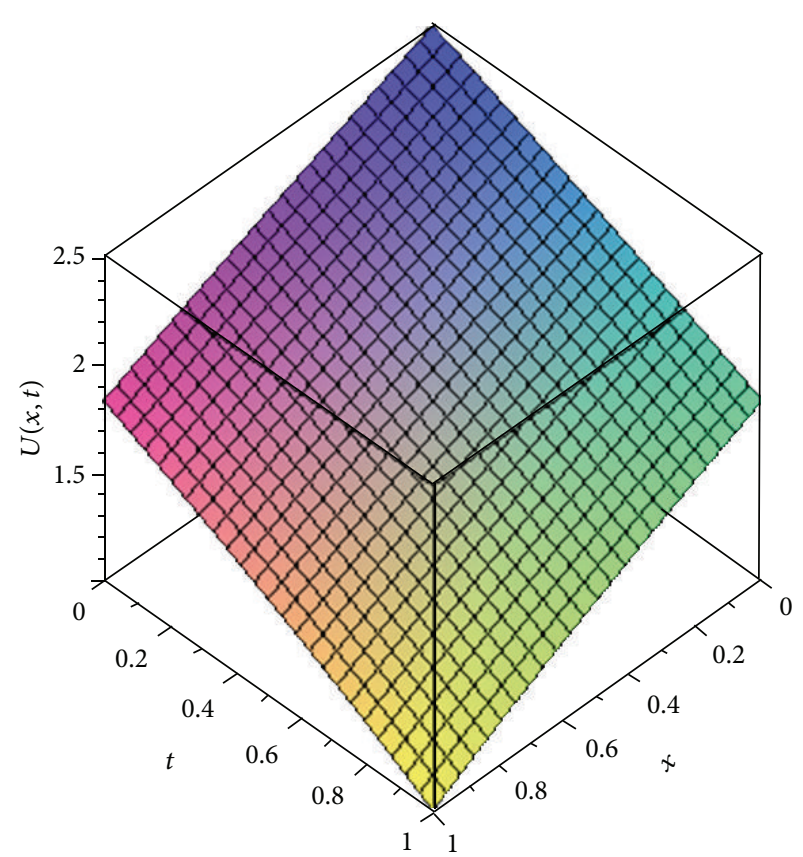

(d)

FIGURE 1: The behaviour of the $U(x, t)$ with respect to $x$ and $t$ being obtained, with (a) $\alpha=1 / 3$; (b) $\alpha=1 / 2$; (c) $\alpha=1$; (d) exact solution.

Next, we decompose the unknown function $U(x, t)$ into sum of an infinite number of components given by the decomposition series

$$
U=\sum_{n=0}^{\infty} U_{n}
$$

and the nonlinear term can be decomposed as

$$
N U=\sum_{n=0}^{\infty} A_{n},
$$

where $A_{n}$ are Adomian polynomials that are given by

$$
A_{n}=\frac{1}{n !}\left[\frac{d^{n}}{d \lambda^{n}} N\left(\sum_{i=0}^{n} \lambda^{i} U_{i}\right)\right]_{\lambda=0}, \quad n=0,1,2, \ldots
$$




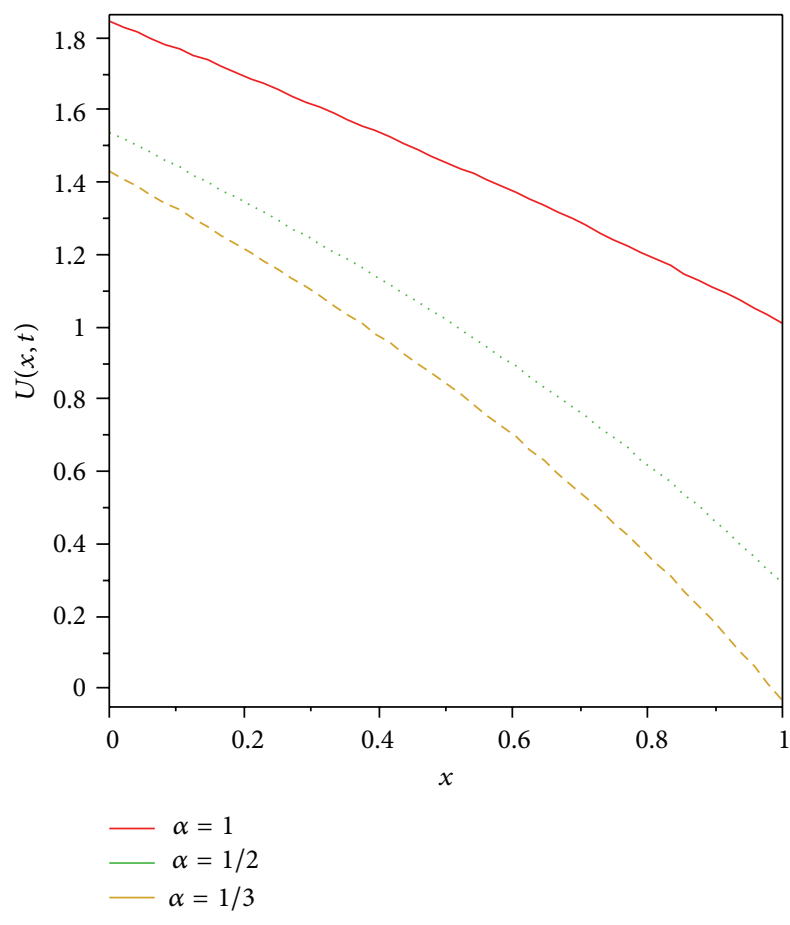

Figure 2: Plots of $U(x, t)$ versus $x$ at $t=1$ for different values of $\alpha$.

The components $U_{0}, U_{1}, U_{2}, \ldots$ are determined recursively by substituting (33) and (34) into (32) leading to

$$
\begin{aligned}
\sum_{n=0}^{\infty} U_{n}= & \sum_{k=0}^{m-1}\left(\frac{\partial^{k} U}{\partial t^{k}}\right)_{t=0} \frac{t^{k}}{k !}+J_{t}^{\alpha} g(x, t) \\
& -J_{t}^{\alpha}\left[R\left(\sum_{n=0}^{\infty} U_{n}\right)+\sum_{n=0}^{\infty} A_{n}\right] .
\end{aligned}
$$

This can be written as

$$
\begin{aligned}
& U_{0}+U_{1}+U_{2}+\cdots \\
& =\sum_{k=0}^{m-1}\left(\frac{\partial^{k} U}{\partial t^{k}}\right)_{t=0} \frac{t^{k}}{k !}+J_{t}^{\alpha} g(x, t) \\
& \quad-J_{t}^{\alpha}\left[R\left(U_{0}+U_{1}+U_{2}+\cdots\right)+\left(A_{0}+A_{1}+A_{2}+\cdots\right)\right] .
\end{aligned}
$$

Adomian method uses the formal recursive relations as

$$
\begin{aligned}
& U_{0}=\sum_{k=0}^{m-1}\left(\frac{\partial^{k} U}{\partial t^{k}}\right)_{t=0} \frac{t^{k}}{k !}+J_{t}^{\alpha} g(x, t), \\
& U_{n+1}=-J_{t}^{\alpha}\left[R\left(U_{n}\right)+A_{n}\right], \quad n \geq 0 .
\end{aligned}
$$

5.2. Solution of the Problem. To solve the nonlinear timefractional Harry Dym equation (22)-(23), we apply the operator $J_{t}^{\alpha}$ on both sides of (22) and use result (9) to obtain

$$
U=\sum_{k=0}^{1-1} \frac{t^{k}}{k !}\left[D_{t}^{k} U\right]_{t=0}+J_{t}^{\alpha}\left[U^{3} D_{x}^{3} U\right] .
$$

This gives the following recursive relations using (38):

$$
\begin{gathered}
U_{0}=\sum_{k=0}^{0} \frac{t^{k}}{k !}\left[D_{t}^{k} U\right]_{t=0}, \\
U_{n+1}=J_{t}^{\alpha}\left[A_{n}\right], \quad n=0,1,2, \ldots,
\end{gathered}
$$

where

$$
\sum_{n=0}^{\infty} A_{n}(U)=U^{3} D_{x}^{3} U
$$

The first few components of Adomian polynomials are given by

$$
\begin{aligned}
A_{0}(U)= & U_{0}^{3} D_{x}^{3} U_{0}, \\
A_{1}(U)= & U_{0}^{3} D_{x}^{3} U_{1}+3 U_{0}^{2} U_{1} D_{x}^{3} U_{0}, \\
A_{2}(U)= & U_{0}^{3} D_{x}^{3} U_{2}+3 U_{0}^{2} U_{1} D_{x}^{3} U_{1} \\
& +\left(3 U_{0} U_{1}^{2}+3 U_{0}^{2} U_{2}\right) D_{x}^{3} U_{0},
\end{aligned}
$$

The components of the solution can be easily found by using the previous recursive relations as

$$
\begin{aligned}
U_{0}(x, t)= & \left(a-\frac{3 \sqrt{b}}{2} x\right)^{2 / 3} \\
U_{1}(x, t)= & -b^{3 / 2}\left(a-\frac{3 \sqrt{b}}{2} x\right)^{-1 / 3} \frac{t^{\alpha}}{\Gamma(\alpha+1)}, \\
U_{2}(x, t)= & -\frac{b^{3}}{2}\left(a-\frac{3 \sqrt{b}}{2} x\right)^{-4 / 3} \frac{t^{2 \alpha}}{\Gamma(2 \alpha+1)} \\
U_{3}(x, t)= & b^{9 / 2}\left(a-\frac{3 \sqrt{b}}{2} x\right)^{-7 / 3} \\
& \times\left(\frac{15}{2} \frac{\Gamma(2 \alpha+1)}{2(\Gamma(\alpha+1))^{2}}-16\right) \frac{t^{3 \alpha}}{\Gamma(3 \alpha+1)}
\end{aligned}
$$

and so on. In this manner the rest of components of the decomposition solution can be obtained. Thus, the ADM 
TABLE 1: Comparison study between HPSTM, ADM, and the exact solution, when $\alpha=1$ and for constant values of $a=4$ and $b=1$.

\begin{tabular}{llccc}
\hline$x$ & $t$ & HPSTM & ADM & Exact solution \\
\hline 0 & 1 & 1.843946953 & 1.843946953 & 1.843946953 \\
0.2 & 1 & 1.694117377 & 1.694117377 & 1.691538112 \\
0.4 & 1 & 1.537581542 & 1.537581542 & 1.534036644 \\
0.6 & 1 & 1.373028020 & 1.373028020 & 1.367980757 \\
0.8 & 1 & 1.198654865 & 1.198654865 & 1.191138425 \\
1.0 & 1 & 1.011880649 & 1.011880649 & 1.000000000 \\
\hline
\end{tabular}

solution $U(x, t)$ of $(22)$ is given as

$$
\begin{aligned}
U(x, t)= & \left(a-\frac{3 \sqrt{b}}{2} x\right)^{2 / 3} \\
& -b^{3 / 2}\left(a-\frac{3 \sqrt{b}}{2} x\right)^{-1 / 3} \frac{t^{\alpha}}{\Gamma(\alpha+1)} \\
& -\frac{b^{3}}{2}\left(a-\frac{3 \sqrt{b}}{2} x\right)^{-4 / 3} \frac{t^{2 \alpha}}{\Gamma(2 \alpha+1)} \\
& +b^{9 / 2}\left(a-\frac{3 \sqrt{b}}{2} x\right)^{-7 / 3} \\
& \times\left(\frac{15}{2} \frac{\Gamma(2 \alpha+1)}{2(\Gamma(\alpha+1))^{2}}-16\right) \frac{t^{3 \alpha}}{\Gamma(3 \alpha+1)}+\cdots,
\end{aligned}
$$

which is the same solution as obtained by using HPSTM.

From Table 1, it is observed that the values of the approximate solution at different grid points obtained by the HPSTM and ADM are close to the values of the exact solution with high accuracy at the third term approximation. It can also be noted that the accuracy increases as the order of approximation increases.

\section{Conclusions}

In this paper, the homotopy perturbation sumudu transform method (HPSTM) and the Adomian decomposition method (ADM) are successfully applied for solving nonlinear timefractional Harry Dym equation. The comparison between the third order terms solution of the HPSTM, ADM, and exact solution is given in Table 1 . It is observed that for $t=1$ and $\alpha=1$, there is a good agreement between the HPSTM, ADM, and exact solution. Therefore, these two methods are very powerful and efficient techniques for solving different kinds of linear and nonlinear fractional differential equations arising in different fields of science and engineering. However, HPSTM has an advantage over the Adomian decomposition method (ADM) such that it solves the nonlinear problems without using Adomian polynomials. In conclusion, the HPSTM may be considered as a nice refinement in existing numerical techniques and might find wide applications.

\section{Acknowledgments}

The authors are very grateful to the referees for their valuable suggestions and comments for the improvement of the paper. The third author also gratefully acknowledges that this research was partially supported by the University Putra Malaysia under the Research Universiti Grant Scheme 05-0109-0720RU and the Fundamental Research Grant Scheme 0111-09-723FR.

\section{References}

[1] G. O. Young, "Definition of physical consistent damping laws with fractional derivatives," Zeitschrift für Angewandte Mathematik und Mechanik, vol. 75, pp. 623-635, 1995.

[2] R. Hilfer, Ed., Applications of Fractional Calculus in Physics, World Scientific, Singapore, 2000.

[3] I. Podlubny, Fractional Differential Equations, Academic Press, New York, NY, USA, 1999.

[4] F. Mainardi, Y. Luchko, and G. Pagnini, "The fundamental solution of the space-time fractional diffusion equation," Fractional Calculus and Applied Analysis, vol. 4, pp. 153-192, 2001.

[5] L. Debnath, "Fractional integrals and fractional differential equations in fluid mechanics," Fractional Calculus and Applied Analysis, vol. 6, pp. 119-155, 2003.

[6] M. Caputo, Elasticita e Dissipazione, Zani-Chelli, Bologna, Italy, 1969.

[7] K. S. Miller and B. Ross, An Introduction to the Fractional Calculus and Fractional Differential Equations, Wiley, New York, NY, USA, 1993.

[8] K. B. Oldham and J. Spanier, The Fractional Calculus Theory and Applications of Differentiation and Integration to Arbitrary Order, Academic Press, New York, NY, USA, 1974.

[9] A. A. Kilbas, H. M. Srivastava, and J. J. Trujillo, Theory and Applications of Fractional Differential Equations, Elsevier, Amsterdam, The Netherlands, 2006.

[10] R. Mokhtari, "Exact solutions of the Harry-Dym equation," Communications in Theoretical Physics, vol. 55, no. 2, pp. 204208, 2011

[11] M. D. Kruskal and J. Moser, Dynamical Systems, Theory and Applications, Lecturer Notes Physics, Springer, Berlin, Germany, 1975.

[12] G. L. Vasconcelos and L. P. Kadanoff, "Stationary solutions for the Saffman-Taylor problem with surface tension," Physical Review A, vol. 44, no. 10, pp. 6490-6495, 1991.

[13] F. Gesztesy and K. Unterkofler, "Isospectral deformations for Strum-Liouville and Dirac-type operators and associated nonlinear evolution equations," Reports on Mathematical Physics, vol. 31, no. 2, pp. 113-137, 1992. 
[14] S. Kumar, M. P. Tripathi, and O. P. Singh, "A fractional model of Harry Dym equation and its approximate solution," Ain Shams Engineering Journal, vol. 4, no. 1, pp. 111-115, 2013.

[15] J. H. He, "Homotopy perturbation technique," Computer Methods in Applied Mechanics and Engineering, vol. 178, no. 3-4, pp. 257-262, 1999.

[16] J. H. He, "Homotopy perturbation method: a new nonlinear analytical technique," Applied Mathematics and Computation, vol. 135, no. 1, pp. 73-79, 2003.

[17] J. H. He, "Asymptotic methods for solitary solutions and compactions," Abstract and Applied Analysis, vol. 2012, Article ID 916793, 130 pages, 2012.

[18] D. D. Ganji, “The applications of He's homotopy perturbation method to nonlinear equation arising in heat transfer," Physics Letters A, vol. 335, pp. 337-341, 2006.

[19] D. D. Ganji and M. Rafei, "Solitary wave solutions for a generalized Hirota-Satsuma coupled KdV equation by homotopy perturbation method," Physics Letters A, vol. 356, no. 2, pp. 131137, 2006.

[20] A. Yildirim, "An algorithm for solving the fractional nonlinear Schrödinger equation by means of the homotopy perturbation method," International Journal of Nonlinear Sciences and Numerical Simulation, vol. 10, no. 4, pp. 445-450, 2009.

[21] N. H. Sweilam and M. M. Khader, "Exact solutions of some coupled nonlinear partial differential equations using the homotopy perturbation method," Computers and Mathematics with Applications, vol. 58, no. 11-12, pp. 2134-2141, 2009.

[22] M. M. Rashidi, D. D. Ganji, and S. Dinarvand, "Explicit analytical solutions of the generalized burger and burgerfisher equations by homotopy perturbation method," Numerical Methods for Partial Differential Equations, vol. 25, no. 2, pp. 409-417, 2009.

[23] A. Yildirim, "He's homotopy perturbation method for nonlinear differential-difference equations," International Journal of Computer Mathematics, vol. 87, no. 5, pp. 992-996, 2010.

[24] H. Jafari, A. M. Wazwaz, and C. M. Khalique, "Homotopy perturbation and variational iteration methods for solving fuzzy differential equations," Communications in Fractional Calculus, vol. 3, no. 1, pp. 38-48, 2012.

[25] A. Ghorbani and J. Saberi-Nadjafi, "He’s homotopy perturbation method for calculating adomian polynomials," International Journal of Nonlinear Sciences and Numerical Simulation, vol. 8, no. 2, pp. 229-232, 2007.

[26] A. Ghorbani, "Beyond Adomian polynomials: he polynomials," Chaos, Solitons and Fractals, vol. 39, no. 3, pp. 1486-1492, 2009.

[27] S. A. Khuri, "A Laplace decomposition algorithm applied to a class of nonlinear differential equations," Journal of Applied Mathematics, vol. 1, no. 4, pp. 141-155, 2001.

[28] M. Khan and M. Hussain, "Application of Laplace decomposition method on semi-infinite domain," Numerical Algorithms, vol. 56, no. 2, pp. 211-218, 2011.

[29] M. Khan, M. A. Gondal, and S. Kumar, "A new analytical solution procedure for nonlinear integral equations," Mathematical and Computer Modelling, vol. 55, pp. 1892-1897, 2012.

[30] M. A. Gondal and M. Khan, "Homotopy perturbation method for nonlinear exponential boundary layer equation using Laplace transformation, He's polynomials and pade technology," International Journal of Nonlinear Sciences and Numerical Simulation, vol. 11, no. 12, pp. 1145-1153, 2010.

[31] J. Singh, D. Kumar, and Sushila, "Homotopy perturbation sumudu transform method for nonlinear equations," Advances in Theoretical and Applied Mechanics, vol. 4, pp. 165-175, 2011.
[32] D. Kumar, J. Singh, and S. Rathore, "Sumudu decomposition method for nonlinear equations," International Mathematical Forum, vol. 7, no. 11, pp. 515-521, 2012.

[33] G. K. Watugala, "Sumudu transform-a new integral transform to solve differential equations and control engineering problems," Mathematical Engineering in Industry, vol. 6, no. 4, pp. 319-329, 1998.

[34] S. Weerakoon, "Applications of sumudu transform to partial differential equations," International Journal of Mathematical Education in Science and Technology, vol. 25, no. 2, pp. 277-283, 1994.

[35] S. Weerakoon, "Complex inversion formula for sumudu transforms," International Journal of Mathematical Education in Science and Technology, vol. 29, no. 4, pp. 618-621, 1998.

[36] M. A. Asiru, "Further properties of the sumudu transform and its applications," International Journal of Mathematical Education in Science and Technology, vol. 33, no. 3, pp. 441-449, 2002.

[37] A. Kadem, "Solving the one-dimensional neutron transport equation using Chebyshev polynomials and the sumudu transform," Analele Universitatii din Oradea, vol. 12, pp. 153-171, 2005.

[38] A. Kılıçman, H. Eltayeb, and K. A. M. Atan, "A note on the comparison between laplace and sumudu transforms," Bulletin of the Iranian Mathematical Society, vol. 37, no. 1, pp. 131-141, 2011.

[39] A. Kılıçman and H. E. Gadain, "On the applications of Laplace and Sumudu transforms," Journal of the Franklin Institute, vol. 347, no. 5, pp. 848-862, 2010.

[40] H. Eltayeb, A. Kılıçman, and B. Fisher, "A new integral transform and associated distributions," Integral Transforms and Special Functions, vol. 21, no. 5, pp. 367-379, 2010.

[41] A. Kılıçman and H. Eltayeb, "A note on integral transforms and partial differential equations," Applied Mathematical Sciences, vol. 4, no. 1-4, pp. 109-118, 2010.

[42] A. Kılıçman, H. Eltayeb, and R. P. Agarwal, "On sumudu transform and system of differential equations," Abstract and Applied Analysis, vol. 2010, Article ID 598702, 11 pages, 2010.

[43] J. Zhang, "A sumudu based algorithm for solving differential equations," Computer Science Journal of Moldova, vol. 15, pp. 303-313, 2007.

[44] V. B. L. Chaurasia and J. Singh, "Application of sumudu transform in schödinger equation occurring in quantum mechanics," Applied Mathematical Sciences, vol. 4, no. 57-60, pp. 2843-2850, 2010.

[45] S. T. Mohyud-Din, M. A. Noor, and K. I. Noor, “Traveling wave solutions of seventh-order generalized KdV equations using he's polynomials," International Journal of Nonlinear Sciences and Numerical Simulation, vol. 10, no. 2, pp. 227-233, 2009.

[46] K. Abbaoui and Y. Cherruault, "New ideas for proving convergence of decomposition methods," Computers and Mathematics with Applications, vol. 29, no. 7, pp. 103-108, 1995.

[47] G. Adomian, Solving Frontier Problems of Physics: the Decomposition Method, Kluwer Academic Publishers, Boston, Mass, USA, 1994.

[48] Z. Odibat and S. Momani, "Numerical methods for nonlinear partial differential equations of fractional order," Applied Mathematical Modelling, vol. 32, no. 1, pp. 28-39, 2008. 


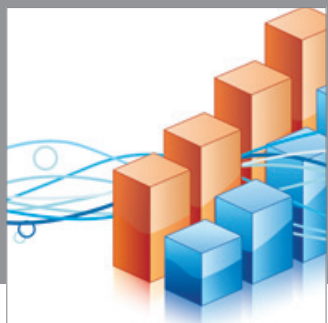

Advances in

Operations Research

mansans

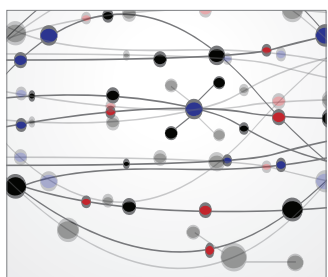

The Scientific World Journal
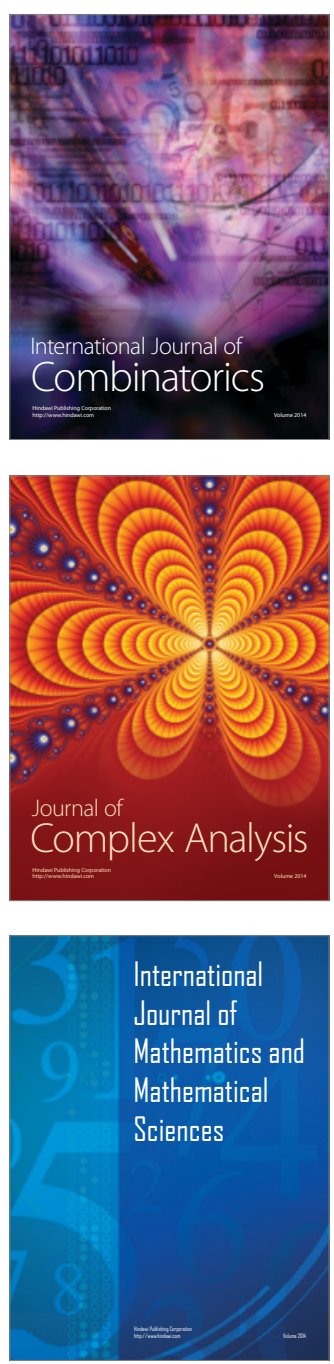
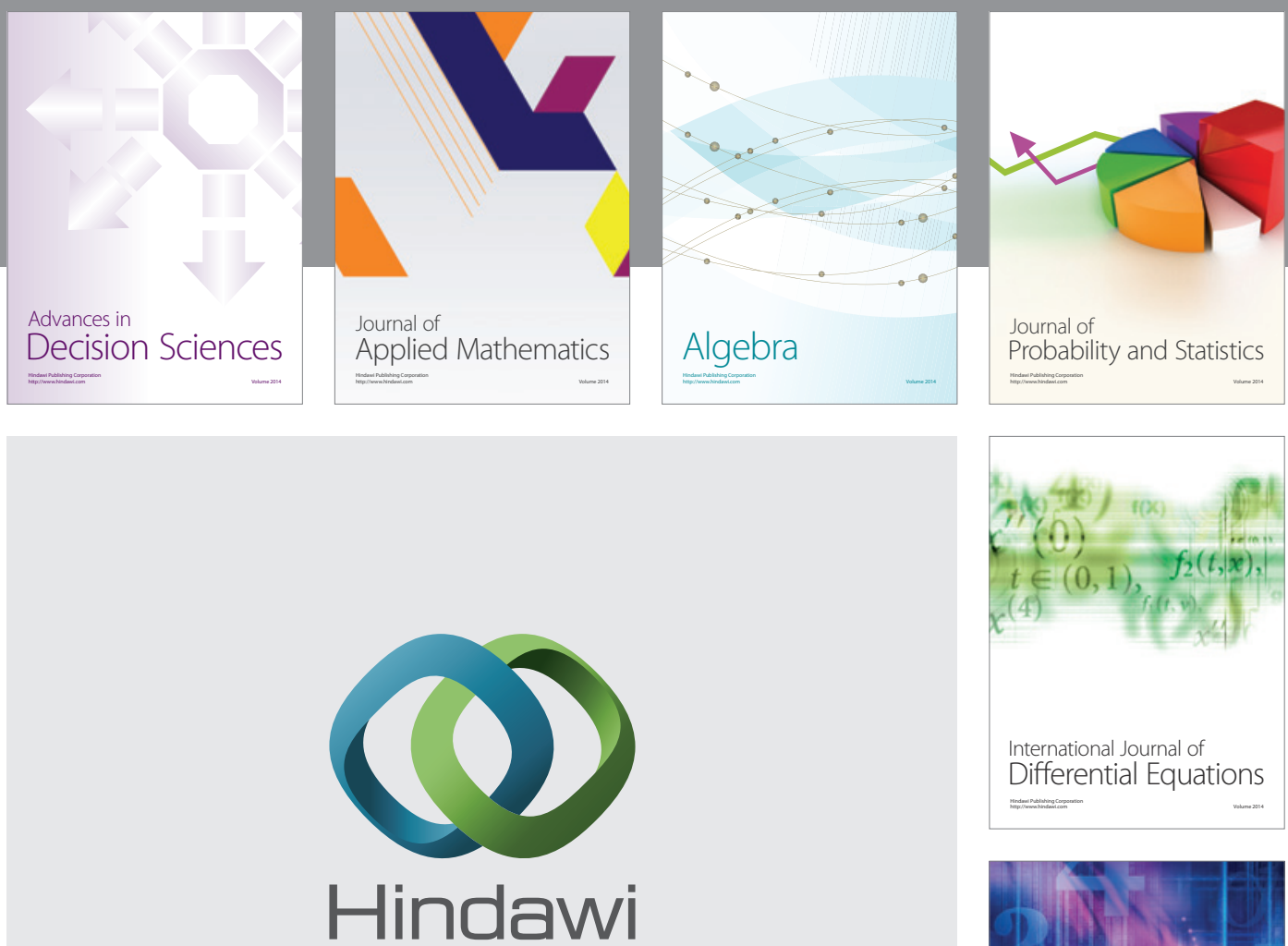

Submit your manuscripts at http://www.hindawi.com
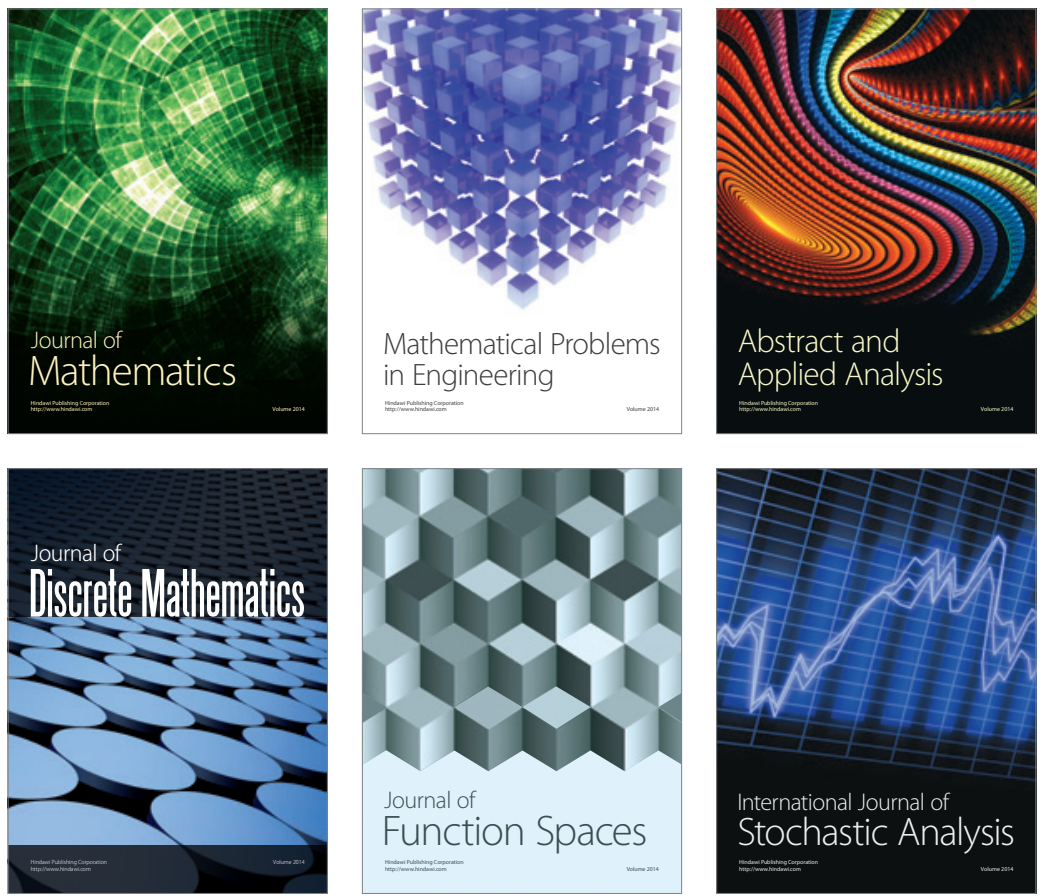

Journal of

Function Spaces

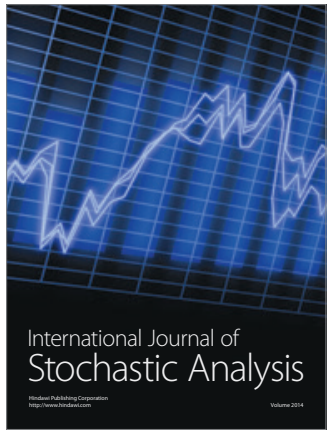

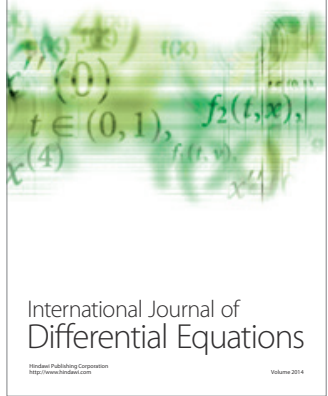
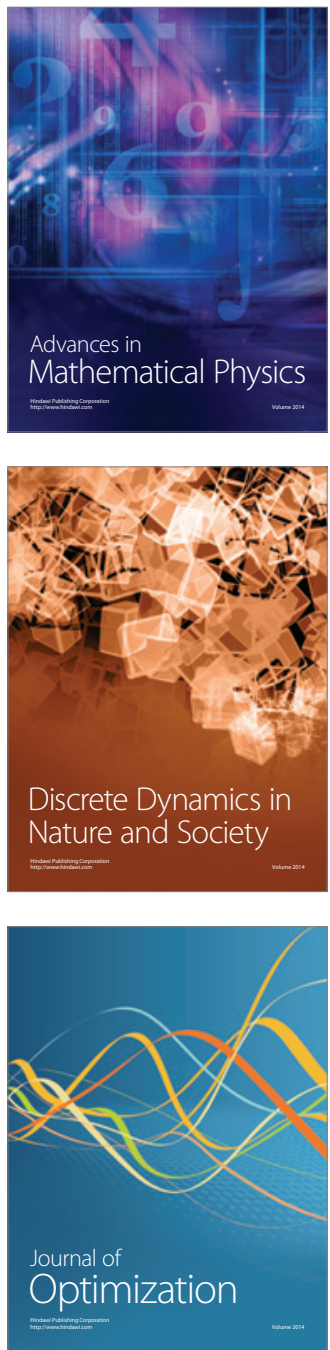